\title{
NOVA OUTBURSTS ON ROTATING OBLATE WHITE DWARFS
}

\author{
A. D. SCOTT
}

Department of Physics, Keele University, Keele, ST5 $5 B G, U K$

\section{Introduction}

Observations of spatially resolved nova shells and reconstructions from emission line profiles show that a ring/blob asymmetry in the ejecta of novae is very common (Martin 1989). The polar blobs expand more rapidly than the equatorial rings, giving the ejecta an overall prolate geometry.

Observations of the formation of carbonaceous and silicate dusts in the same nova provide unambiguous evidence that abundance gradients exist in the ejecta. Carbonaceous dusts form where carbon is more abundant than oxygen and vice versa for silicates (Evans 1990; Gehrz et al. 1992; Smith, Aitken \& Roche 1994; Snijders et al. 1987).

\section{Proposition - shaping of ejecta}

1. Since the polar radius of an oblate white dwarf is less than the equatorial radius, the effective surface gravity and the pressure at the coreenvelope interface at the equator is less than at the poles. The electron degeneracy at the base of the accreted envelope and hence the peak temperature of the thermonuclear runaway (TNR) will therefore be stronger at the poles, all other things being equal (Livio 1992). Where the peak temperature is higher, the violence of the TNR outburst is stronger. Hence material will be ejected at higher velocities in the polar regions than at the equatorial regions, producing prolate ejecta.

2. The larger effective gravity and smaller white dwarf radius at the poles implies that the critical point in stellar wind theory is located at a smaller radius at the poles than at the equator. Hence the terminal wind velocities will be greater at the poles than at the equator, which again produces prolate ejecta (Kato \& Hachisu 1994, for example). 
3. The nova wind is expanding while rotating. The polar region is able to expand freely while the equatorial flow feels a progressively stronger centrifugal barrier (Shore 1992). This follows from the de Laval nozzle analogy used in stellar wind theory. Centrifugal forces act to partially counter gravitation toward the equator; the equatorial wind will therefore be slower than the polar wind.

\section{Proposition - abundance gradients}

Evolutionary calculations of the TNR off ONeMg white dwarfs of different masses (Starrfield et al. 1992) show that the $\mathrm{C} / \mathrm{O}$ ratio increases with the degree of electron degeneracy at the base of the envelope at the start of the TNR. It is therefore proposed that the $\mathrm{C} / \mathrm{O}$ abundance ratio is greater in the polar blobs than in the equatorial rings.

Thus, for the first time, the shape of the nova ejecta and the abundance gradients can be explained simultaneously as a consequence of the same phenomenon, i.e. the rotation and distortion of the white dwarf.

Observational investigations, whether of the underlying binary system or the resolved ejected shell of the nova, are now needed to test this hypothesis. Theoretical investigations, combining hydrodynamical simulations of the nova outburst with models of nucleosynthesis and energy production as a function of latitude on the white dwarf need to be constructed.

Acknowledgements. I thank Professor A. Evans, my thesis supervisor, for his support and encouragement, and Dr. T. Shahbaz for getting me interested in rotating white dwarfs. I also thank Drs. W. Welsh and H. W. Duerbeck for useful discussions. The STARLINK project and the Department of Physics at the University of Keele are thanked for their provision of facilities.

\section{References}

Evans, A., 1990, in "Physics of Classical Novae" Proc. IAU Coll. 122, eds Cassatella, A., Viotti, R., Springer-Verlag, p252

Gehrz, R.D., Jones, T.J., Woodward, C.E., et al., 1992, Ap. J., 400, 671

Kato, M., Hachisu, I., 1994, Ap. J., 437, 802

Livio, M., 1992, Ap. J., 393, 516

Martin, P.G., 1989, in "Classical Novae", eds Bode, M. F., Evans A., Chichester: Wiley \& Sons, p73

Shore, S.N., 1992, An Introduction to Astrophysical Hydrodynamics, San Diego: Academic Press

Smith, C.H., Aitken, D.K., Roche, P.F., 1994, MNRAS, 267, 225

Snijders, M.A.J., Batt, T.J., Roche, P.F., et al. 1987, MNRAS, 228, 329

Starrfield, S., Shore, S.N., Sparks, W.M., et al., 1992, Ap. J., 391, L71 